\title{
The use of highly active retroviral therapy to reduce HIV incidence at the population level
}

\author{
Aranka Anema MSc, Evan Wood MD PhD, Julio S.G. Montaner MD
}

I $\mathrm{n}$ this article, we will argue that the widespread use of highly active antiretroviral therapy has the potential to reduce the incidence of $\mathrm{HIV}$ at the individual and population level (Table 1). However, this approach has been overlooked by public health specialists as a viable prevention strategy. Other practical interventions such as education and the use of condoms and clean needles have helped to reduce the transmission of HIV worldwide. Once proven effective, safe and culturally accepted, biomedical interventions such as vaccines, topical microbicides, pre- and post-exposure prophylaxis and male circumcision offer further potential to prevent HIV transmission. Despite such advances, the global number of new HIV infections continues to increase at an alarming rate. It is therefore timely to explore other possible means to curb the growth of the epidemic.

Clinicians and researchers have long recognized the role that highly active antiretroviral therapy can play in preventing HIV/AIDS. High-quality clinical trials have demonstrated that highly active antiretroviral therapy successfully prevents mother-to-child transmission by lowering maternal plasma concentrations of HIV type 1 (HIV-1) RNA. ${ }^{1}$ As a consequence of widespread access to highly active antiretroviral therapy, mother-to-child transmission of HIV in industrialized nations has become exceedingly rare. ${ }^{2}$ Observational studies have also reported that this therapy can decrease the risk of HIV transmission from percutaneous exposure and between serodiscordant couples by reducing the concentration of HIV RNA in infected people. ${ }^{2}$

Highly active antiretroviral therapy has also been shown to reduce the concentration of HIV-1 RNA in semen, the rectum and the female genital tract. ${ }^{2}$ Furthermore, in a major study from Uganda, no cases of HIV transmission were identified among couples in which the index case had a level of HIV-1 RNA less than 1500 copies/mL. ${ }^{2}$ A dose-response effect was also reported in a Thai study, in which no cases of HIV transmission were observed if the index case's serum level of HIV-1 RNA was less than 1100 copies/mL. ${ }^{3}$ Finally, an observational study of serodiscordant couples in Spain showed that no HIV seroconversions took place in the sexual partners of patients who had received highly active antiretroviral therapy. ${ }^{4}$ This therapy was independently associated with an $80 \%$ reduction in HIV transmission. ${ }^{4}$

At the population level, access to highly active antiretroviral therapy has been temporally associated with substantial reductions in HIV incidence. ${ }^{5}$ For example, populationbased research in Taiwan found a 53\% (95\% confidence interval [CI] 31\%-65\%) reduction in new positive HIV test results after the introduction of free access to highly active

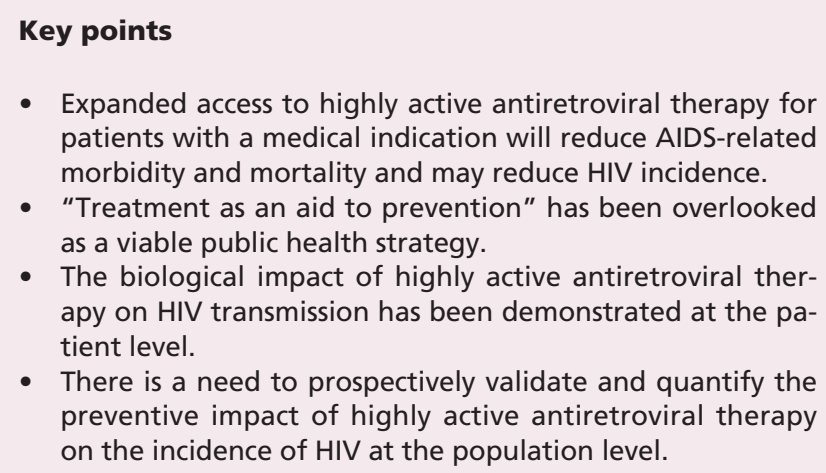

- Expanded access to highly active antiretroviral therapy for patients with a medical indication will reduce AIDS-related morbidity and mortality and may reduce HIV incidence.

- "Treatment as an aid to prevention" has been overlooked as a viable public health strategy.

- The biological impact of highly active antiretroviral therapy on HIV transmission has been demonstrated at the patient level.

- There is a need to prospectively validate and quantify the preventive impact of highly active antiretroviral therapy on the incidence of HIV at the population level.

antiretroviral therapy. ${ }^{6}$ There was no change in the rate of syphilis infections, which was used as a surrogate marker for sexual risk behaviour during the study. In Uganda, expanded access to all clinically eligible people was projected to decrease HIV incidence by $11.2 \%$ (interquartile range $1.8 \%-21.4 \%){ }^{7}$ In Canada, new yearly HIV infections in British Columbia decreased by about $50 \%$ after the introduction of highly active antiretroviral therapy between 1995 and 1998 and has remain unchanged since then despite a noticeable increase in the rate of syphilis infections. ${ }^{5}$ It has been also been suggested that the preventive impact of expanded access to highly active antiretroviral therapy increases along with the number of people treated.

The use of "treatment as an aid to prevention" is not new in the context of public health management of infectious diseases. For example, treatment of pulmonary tuberculosis and recurrent genital herpes prevents disease progression and reduces the risk of transmission. ${ }^{8}$ However, public health policy-makers and program managers have been reluctant to accept this strategy as viable for preventing the growth of the HIV/AIDS epidemic. Critics have argued that the preventive impact of expanded access to highly active antiretroviral therapy may be overridden by increases in HIV risk behaviours. This notion is supported by an ecological study that found widespread access to this therapy among men who have sex with men did not reduce HIV incidence and was associated with elevated risk behaviours. ${ }^{9}$ However, a recent metaanalysis evaluating the impact of highly active antiretroviral therapy use on sexual behaviours found no statistically significant increase in sexual risk behaviours among people receiving treatment (odds ratio $0.92,95 \%$ CI 0.65-1.31). ${ }^{10}$

From the BC Centre for Excellence in HIVIAIDS and the Department of Medicine, University of British Columbia, Vancouver, BC 
Table 1: Potential challenges and successes of expanded access to highly active antiretroviral therapy to all people with a medical indication

Potential challenges

Potential successes

- Limited knowledge of HIV status among infected people

- Extensive and innovative strategies for vulnerable and hardto-reach populations

- Increases in risk behaviour while receiving highly active antiretroviral therapy (e.g., treatment optimism, risk compensation)

- Incomplete adherence to highly active antiretroviral therapy

- Increases in population-level drug resistance and adverse events

- Increased short-term health care costs
- Decreased AIDS-related morbidity

- Decreased AIDS-related mortality

- Reduced HIV incidence

- Reduced HIV prevalence in the long term

- Intermediate and long-term decrease in health care costs

- Health care cost savings because of fewer HIV infections
Some have argued that successful treatment is too difficult for patients to initiate at a comparatively early stage of their illness. Indeed, we agree that people with HIV should be encouraged to initiate treatment only when clinically eligible. Yet even though some infections occur in the acute stages of a patient's infection, often before HIV infection is diagnosed, many exposures occur when patients are already aware of their serostatus and when patients are eligible for therapy. Furthermore, the reality is that highly active antiretroviral therapy coverage in Canada is suboptimal. It is estimated that over 50\% of Canadians infected with HIV access treatment either late or not at all. Thus, increasing access to highly active antiretroviral therapy in Canada will result in decreased AIDS-related morbidity and mortality as well as potentially fewer infections.

Concerns have also been raised to suggest that treatment as an aid to prevention might cause a rise in population-level viral drug resistance, thus reducing the preventive benefit of highly active antiretroviral therapy on HIV transmission. Yet, population-based mathematical modelling suggests that expanded access would only be associated with a small increase in population-level drug resistance and would therefore have a limited adverse impact on HIV transmission. ${ }^{11}$ Adding to this finding, studies suggest that transmission of HIV from people with drug-resistant viral strains to serodiscordant contacts is reduced compared with people with wild-type HIV strains. ${ }^{12}$

The Swiss National AIDS Commission recently put forward a statement suggesting that people with HIV who adhere to highly active antiretroviral therapy, who have undetectable viral loads and who have no sexually transmitted infections are not infectious..$^{13}$ As a result, the need to prospectively validate and quantify the effect of this therapy on HIV prevention has grown substantially. Treatment as an aid to prevention should be explored in diverse settings, including in developed and developing countries. There are several research strategies that could help inform this debate. For instance, serodiscordant couples could be randomized to initiate highly active antiretroviral therapy at different CD4 thresholds to determine if greater periods of plasma viral load suppression are related to reduced HIV transmission. Community-based trials could also be used to measure longitudinal patterns of plasma HIV RNA and HIV incidence associated with expanded uptake of highly active antiretroviral therapy. Evidence derived from these research efforts will decrease AIDS-related morbidity and mortality and inform policy-makers about the role of treatment as an aid to prevention.

This article has been peer reviewed.

Competing interests: None declared.

Contributors: Aranka Anema contributed to the conception and design of the manuscript, and wrote the first draft. Evan Wood contributed to the conception and design of the manuscript. Julio Montaner conceived of the "treatment as an aid to prevention" hypothesis. Each of the authors revised the manuscript for important intellectual content and approved the final version submitted for publication.

\section{REFERENCES}

1. Brocklehurst P, Volmink J. Antiretrovirals for reducing the risk of mother-to-child transmission of HIV infection. Cochrane Database Syst Rev 2002;(2)CD003510.

2. Cohen MS, Gay C, Kashuba AD, et al. Narrative review: antiretroviral therapy to prevent the sexual transmission of HIV-1. Ann Intern Med 2007;146:591-601.

3. Tovanabutra S, Robison V, Wongtrakul J, et al. Male viral load and heterosexual transmission of HIV-1 subtype E in northern Thailand. J Acquir Immune Defic Syndr 2002;3:275-83.

4. Castilla J, Del Romero J, Hernando V, et al. Effectiveness of highly active antiretroviral therapy in reducing heterosexual transmission of HIV. J Acquir Immune Defic Syndr 2005;40:96-101.

5. Montaner JSG, Hogg RS, Wood E, et al. The case for expanding access to highly active anti-retroviral therapy to curb the growth of the HIV epidemic. Lancet 2006;368:531-6.

6. Fang CT, Hsu HM, Twu SJ, et al. Decreased HIV transmission after a policy of providing free access to highly active antiretroviral therapy in Taiwan. J Infect Dis 2004; 190:879-85.

7. Abbas UL, Anderson RM, Mellors JW. Potential impact of antiretroviral therapy on HIV-1 transmission and AIDS mortality in resource-limited settings. J Acquir Immune Defic Syndr 2006;41:632-41.

8. Martinez V, Caumes E, Chosidow O. Treatment to prevent recurrent genital herpes. Curr Opin Infect Dis 2008;21:42-8.

9. Katz MH, Schwarcz SK, Kellogg TA, et al. Impact of highly active antiretroviral treatment on HIV seroincidence among men who have sex with men: San Francisco. Am J Public Health 2002;92:388-94.

10. Crepaz N, Hart TA, Marks G. Highly active antiretroviral therapy and sexual risk behavior: a meta-analytic review. JAMA 2004;292:224-36.

11. Lima VD, Hogg RS, et al. Expanded access to highly active antiretroviral therapy: a potentially powerful strategy to curb the growth of the HIV epidemic. J Infect Dis. In press.

12. Yerly S, Jost S, Telenti A, et al. Infrequent transmission of HIV-1 drug-resistant variants. Antivir Ther 2004;9:375-84

13. Vernazza P, Hirschel B, Bernasconi E, et al. Les personnes séropositives ne souffrant d'aucune autre MST et suivant un traitement antirétroviral efficace ne transmettent pas le VIH par voie sexuelle. Bulletin des médecins suisses 2008;89:165-9.

Correspondence to: Dr. Julio Montaner, BC Centre for Excellence in HIV/AIDS, Rm. 667, 667-1081 Burrard St., Vancouver BC

V6Z 1Y6; fax 604 806-8527; jmontaner@cfenet.ubc.ca 